Case Report

\title{
Idiopathic Hypertrophic Pachymeningitis: A Case Report from Bangladesh
}

\author{
Meera Momtaz Sabeka', Md. Nazrul Islam² \\ ${ }^{1}$ Registrar, Department of Neurophysiology, National Institute of Neurosciences \& Hospital, Dhaka, Bangladesh; \\ ${ }^{2}$ Assistant Professor, Department of Neuroradiology \& Imaging, National Institute of Neurosciences \& \\ Hospital, Dhaka, Bangladesh
}

[Received: 2 February 2017; Revised: 18 March 2017; Accepted: 21 May 2017; Published: 1 July 2017]

\begin{abstract}
A 52 years old lady presented with sudden severe headache with the history of similar intense headache twenty years back which ended up with left sided blindness. Her physical examination was unremarkable except optic atrophy of the left eye. Investigation included biochemical work up, imaging studies and CSF study. The MRI of brain with contrast gave the key diagnostic clue with characteristic findings of hypertrophic pachymeningitis. Other investigations helped to rule out possible etiologies and the diagnosis idiopathic hypertrophic pachymeningitis was finally made. The patient has been treated with steroid and enjoyed improvement in her yearlong symptoms. [Journal of National Institute of Neurosciences Bangladesh, 2017;3(2): 110-112]
\end{abstract}

Keywords: IHP, Meningitis, Headache

Correspondence: Dr. Meera Momtaz Sabeka, Registrar, Department of Neurophysiology, National Institute of Neurosciences \& Hospital, Sher-E-Bangla Nagar, Agargaon, Dhaka, Bangladesh; Email: meerasabeka@gmail.com; Cell no.: 01715933417

Contribution to authors: Dr. Meera has clinically diagnosed the case the mange the case. Dr. Meera has written the manuscript. and Dr. Meera has revised the manuscript. Dr. Nazrul diagnosed the case radiologically.

How to cite this article: Sabeka MM, Islam MN. Idiopathic Hypertrophic Pachymeningitis: A Case Report from Bangladesh. J Natl Inst Neurosci Bangladesh, 2017;3(2): 110-112

Copyright: (C2017. Sabeka \& Islam. Published by Journal of National Institute of Neurosciences Bangladesh. This article is published under the Creative Commons CC BY-NC License (https://creativecommons.org/licenses/by-nc/4.0/). This license permits use, distribution and reproduction in any medium, provided the original work is properly cited, and is not used for commercial purposes.

\section{Introduction}

Idiopathic hypertrophic pachymeningitis is a rare and unique clinical entity, characterized by thickening and fibrosis of dura mater. Exact etiopathogenesis of this entity is still unknown, but it is speculated to be an autoimmune phenomenon or occur as a direct result of infectious or infiltrative pathology ${ }^{1}$. The diagnosis of IHP relies on the exclusion of other possible causes of pachymeningitis, such as neurosarcoidosis, neurosyphilis, tuberculosis, rheumatoid pachymeningitis and Wegener's granulomatosis ${ }^{2}$. The condition may sometimes mimic Tolosa Hunt syndrome or hemicranial continua ${ }^{3}$. It can now be broadly divided into two forms, 'primary' or 'idiopathic hypertrophic pachymeningitis' where no identifiable cause is found and 'secondary' where identifiable causes co-exist, although their definite role in disease causation is uncertain 1.
Common clinical features include headaches, multiple cranial nerve palsies and cerebellar dysfunction occurring alone or in combination ${ }^{4}$. Headache is a common symptom, which can be focal or diffuse and at times may be the only symptom for years before other symptoms manifest ${ }^{4}$. The headache possibly denotes dural inflammation, whereas dense fibrous encasement and ischemic damage by hypertrophic tissue is the likely explanation for the cranial nerve deficits. Uncommon clinical presentations include encephalitis, hypo-pituitarism, diabetes inspidus, venous sinus thrombosis and hydrocephalus ${ }^{6}$.

\section{Case Presentation}

A 52 year female, known diabetic was presented with intense, excruciating headache for last one year. Headache involved the left fronto-parietal region, along 
with eye pain, nausea and occasional vomiting. She had history of similar headache 20 years back and became blind in the left side. After becoming blind, her that episode subsided. Thereafter, she did not have any headache or any other complaints in last twenty years. She has no other neurological or systemic complaints and was begging for pain relief. On physical examination she had reduced vision in left eye (no perception of light) and optic atrophy in the same eye and intra-ocular pressure was $10 \mathrm{mmHg}$ in both eyes. Other general and systemic examination was unremarkable. Her investigation report revealed abnormality in brain imaging and subtle changes in some biochemical parameter. Investigations revealed an ESR of $51 \mathrm{~mm}$ and negative ANA, ANCA and RA factor.CSF examination showed absence of pleocytosis, a protein level of 70 $\mathrm{mg} \%$ and normal sugar. Gram's stain, fungal smear and antigen detection and staining for AFB were negative. Serum and CSF VDRL tests were negative. CSF ADA levels were $9.6 \mathrm{U} / \mathrm{L}$. X-ray chest and CT brain were normal and CT of orbit depicted mildly protruded left eye ball. MRI head (plain and contrast) revealed enhancing thick $(1 \mathrm{~cm})$ meninges 4 along left tentorium cerebelli.

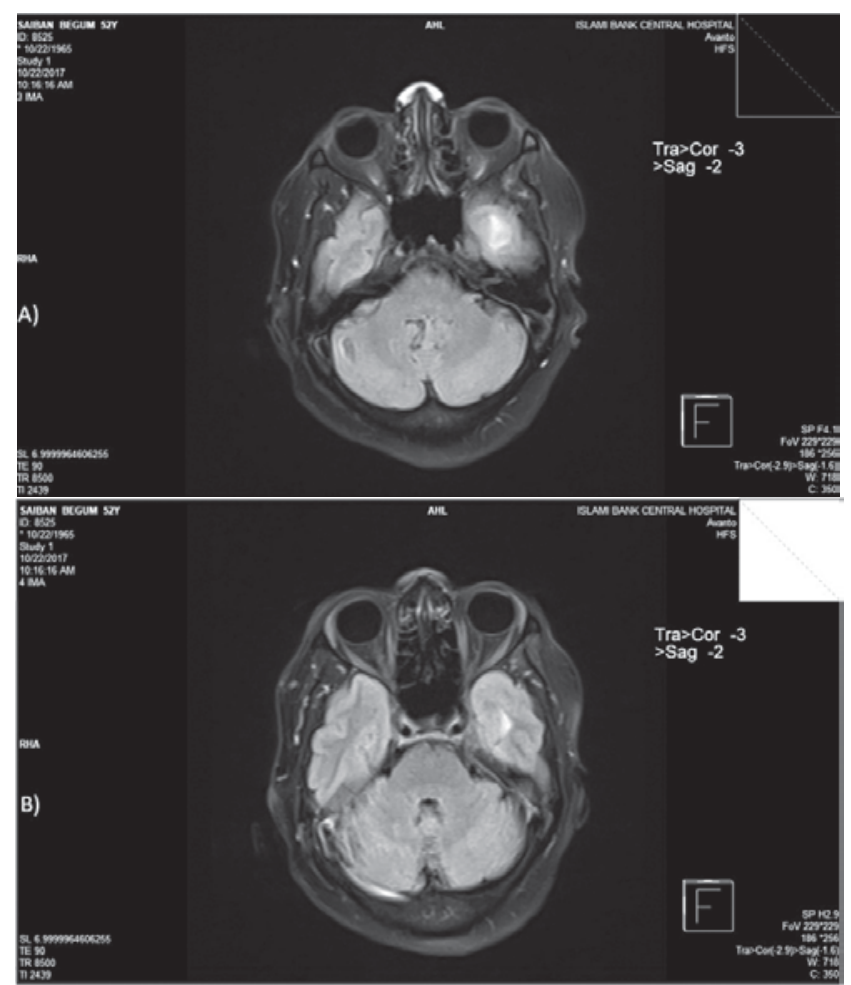

Figure I: Axial FLAIR MRI showing iso-hypointense thickening $(1 \mathrm{~cm})$ of left side of tentorium cerebelli
The lesion was T1hypo, T2 FLAIR hyper intensity and not partially diffusion restricted. Few tiny T2-FLAIR hyper intensity foci are seen in sub-cortical and paraventricular region of both fronto-parietal regions, which are iso-intense in $\mathrm{T} 1$ and are not diffusion restricted (Figure). MRA and MRV of cerebral vessels were normal. Considering the history and results of other investigations, a tentative diagnosis of possible 'idiopathic hypertrophic pachymeningitis' was made and she was started on steroid therapy. Within days she showed a marked improvement in her clinical symptoms.

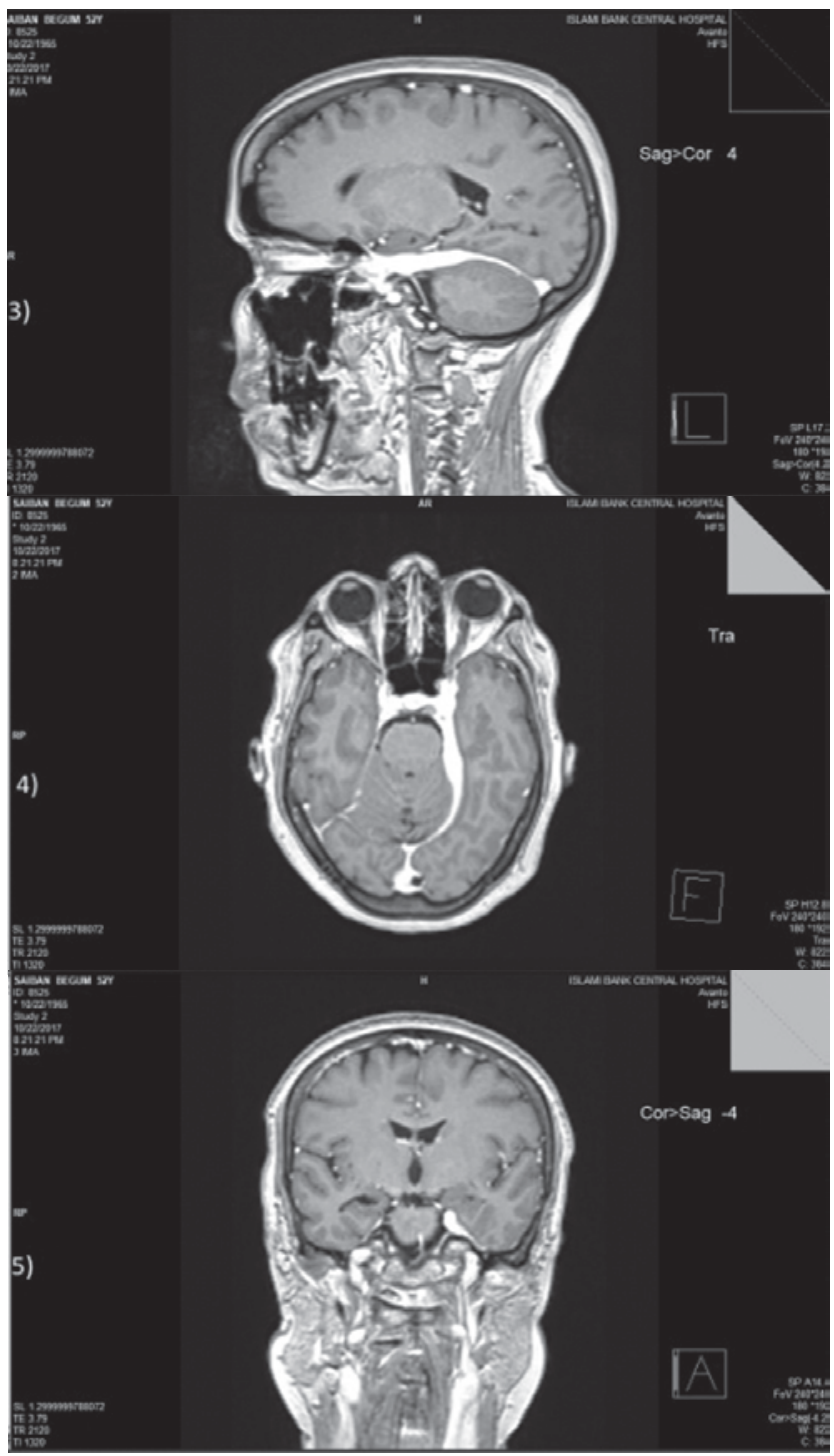

Figure II: Post contrast T1 axial, sagittal \& coronal MRI showing strong enhancement of left side of tentorium cerebelli thickening

\section{Discussion}

The case presented as sudden severe headache with the history of similar intense headache twenty years back 
which ended up with left sided blindness. The physical examination was unremarkable except optic atrophy of the left eye.

Investigative work up of this condition aims at excluding all possible causes of infectious, noninfectious and malignant etiologies. This includes biochemical work up, imaging studies and CSF study. Elevation of ESR is common. Tests like ACE levels, vasculitic profile, Mantoux test and cultures for bacteria and fungi should be done to rule out specific disease states. CSF is an important investigation, but it showed inconclusive results in this case. Although protein levels were moderately elevated, normal sugar level and absence of pleocytosis failed to point towards any infectious process.

Imaging is an important investigation to identify a meningeal based pathology and exclude mass lesions in the brainstem or skull base. The changes on imaging are probably related to the presence of dense fibrous tissue, with decrease in interstitial space and paucity of imageable? free water, accounting for the hypointensity on both $\mathrm{T} 1$ and $\mathrm{T} 2 \mathrm{~W}$ images. Idiopathic hypertrophic cranial pachymeningitis usually involves dura at tentorium cerebelli, cavernous sinus and base of the skull. The extensive involvement at the anterior cranial fossa is extremely rare $^{7}$. Presence of associated leptomeningeal enhancement or parenchymal abnormalities with the exception of brain edema should suggest an alternate diagnosis ${ }^{8-9}$. The initial non-contrast imaging studies in this patient delayed the diagnosis.

A dural biopsy is considered important for differentiating idiopathic from secondary forms of pachymeningitis ${ }^{4}$. Since many associations and secondary causes for pachymeningeal thickening and enhancement exist, pathological examination of meninges is essential. Pathological features include thickening, fibrosis and presence of inflammatory cells including plasma cells and lymphocytes. Presence of granulomas or vasculitis aids in establishing a specific etiology. In the absence of dural biopsy, clinical symptomatology, imaging characteristics, absence of abnormal laboratory and CSF studies, long course of the disease and responsiveness to steroid therapy point towards an idiopathic variety in this case.

Steroid therapy has been considered the mainstay of therapy in this disease. However, the disease progression might continue despite institution of steroids and many patients may eventually become steroid dependent ${ }^{4}$. Considering occult tuberculosis, empirical use of antitubercular therapy has been advocated, however, previous studies did not show any improvement ${ }^{4}$. Surgery has both therapeutic and diagnostic benefits5. When the radiological or laboratory evaluation is uncertain, but neurological deficits are present, a prompt surgical approach should be considered ${ }^{10}$. Postoperative steroid therapy and close observation for recurrence are necessary to ensure a good long-term outcome ${ }^{10}$.

\section{Conclusion}

Thus, hypertrophic pachymeningitis is an important cause of recurrent cranial neuropathies and headaches. Hypertrophic pachymeningitis can be diagnosed with contrast MRI of brain when the clinician maintains a high index of suspicion for this condition. Nearly accurate diagnosis and treatment may alleviate patient's sufferings, and aim of the therapy is to prevent permanent damage to neural structures.

\section{References}

1. Karthik SN, Bhanu K, Velayutham S, Jawahar M. Hypertrophic pachymeningitis. Ann Indian Acad Neurol 2011;14(3): 203-204

2. Yu Chang Lee, Yao Chung Chueng, Shin Wei Hsu and Chun Chung Lui. Idiopathic hypertrophic cranial pachymeningitis: case report with 7 years of imaging follow-up. American Journal of Neuroradiology 2003;24 (1):119-123

3. Russo A, Silvestro M, Cirillo M, Tessitore A, Tedeschi G. Idiopathic hypertrophic pachymeningitis mimicking hemicrania continua: An unusual clinical case. Cephalalgia. 2017:333102417708773

4. Prabhakar S, Bhatia R, Lal V, Singh P. Hypertrophic pachymeningitis: varied manifestations of a single disease entity. Neurol India 2002;50:45-52

5. Hatano N, Behari S, Nagatani T et al. Idiopathic hypertrophic pachymeningitis: clinic-radiological spectrum and therapeutic options. Neurosurgery 1999;45:1336-1344

6. Goyal M, Malik A, Mishra NK, et al. Idiopathic hypertrophic pachymeningitis: spectrum of the disease. Neuroradiology 1997; 39:619-623

7. Boonyawiroj S, Phanthumchinda K. Extensive anterior cranial fossa idiopathic hypertrophic pachymeningitis: a case report and review of the literature. J Med Assoc Thai 2005;88(12):1934-40

8. Kon T, Ishihara N, Kamimura T. Unusual MRI findings of hypertrophic pachymeningitis with irregular dural thickening and severe brain oedema. Eur J Radiology Extra 2004;50:39-43

9. Tuncel D, Yücesan C, Erden E, Savaş A, Erden I, Mutluer N. Idiopathic hypertrophic cranial pachymeningitis with perifocal brain edema. Clin Neurol Neurosurg 2005;107:249-52

10. Huang Y, Chen J, Gui L. A case of idiopathic hypertrophic pachymeningitis presenting with chronic headache and multiple cranial nerve palsies: A case report. Medicine 2017;96(29):e7549 
